\title{
Research on Knowledge Management of Construction Cost Control
}

\author{
Liu Li \\ School of Management, Xi'an University of Architecture and Technology, Xi'an 710055, China \\ 441408079@qq.com
}

\begin{abstract}
In recent years, the construction cost control problems are very prominent, and it is imperative to study the construction cost control. In order to solve the problem of the low efficiency of the case knowledge description and the case retrieval in the field of the current construction cost control, the construction cost control case knowledge management model is proposed to represent cost knowledge. At the same time, the construction cost control case database is set up, and the weighted gray correlation degree method are used to achieve case retrieval, then we can find out the best solution for the new construction cost management control problem. The construction enterprises can realize the knowledge management in the field of construction cost control, so as to strengthen the construction cost control and reduce the cost of the enterprise.
\end{abstract}

Keywords: construction cost; knowledge representation; case retrieval; similarity.

\section{Introduction}

Construction cost control is the most critical aspect in the project management activities. Construction cost control is that the construction enterprises make cost estimates, planning, control, accounting, analysis, evaluation, in order to strictly control the total cost of construction within the program objectives. Construction cost control is to ensure actual costs to meet the requirements of target budget and plans [1]

Currently, the construction cost management in our country has also been on the level of traditional control. Intelligent is at a lower level. Project cost control experience cannot be effectively stored. The cost knowledge cannot be shared. Thus, a comprehensive, systematic, long-term, efficient management method is urgently needed in terms of construction cost control.

In recent years, knowledge management became a hot topic in many areas, knowledge management has also been widely used in construction engineering. Knowledge management is actually a systematic and organized process of dealing with knowledge and information [2].Construction project cost continuous improvement process is to realize the acquisition, sharing, innovation and application of the cost knowledge [3] .

\section{Construction Cost Control Case Management Knowledge Processes}

There are 2 key points in the process of construction cost control case knowledge management. The key point 1 is how to build a construction cost control matrix model of knowledge representation. The key point 2 is how to make a similarity search for cost control cases. We can realize the process of knowledge sharing, innovation and storage in the construction cost control case knowledge representation matrix model by acquiring knowledge and processing the related data. When the new project cost cases appear, according to its characteristic properties, weighted gray correlation coefficient can be used to match construction costs case knowledge database. If it meets the search criteria, similar case information can be retrieved from the case base, and it can provide the basis for the construction companies to solve the current problems. If the match is not successful, the new project cost information will be stored in the knowledge database as a new cost case. Through continuous improvement of the case base, it can be the best solution to meet the new case. 


\section{Database of Construction Cost Control Case and Knowledge Representation}

Case representation is a strict and formal description of the historical experience data, which is based on the corresponding data structure. Extraction and utilization of engineering properties is the basis of the case representation. Existing case representation methods are: logical notation, production notation, frame representation, semantic network representation [4].Among them, frame representation is a general structure of semantic network. It can greatly satisfy the needs of engineering knowledge representation and case reasoning. And it is very easy for the system to carry on the inference, so the framework representation can be widely used [5] .

The important role of the framework is data structure to reflect the characteristics of the case. The sum of all characteristic attributes of the case can be as a framework. Each slot is used to describe the each component content of case. As an important content of slot, each side is used to describe a set of characteristic properties. Its formal description is as follows:

Case Number:

Frame Name :( Case Project Name)

Slot 1. Project Description

Slot 2. Engineering Characterization

Side 1. Engineering Purposes

Side 2. Project Type

Side 3 .Location

.......

Slot 3. Results

Side1. Quantity Equipped

Side2. Construction Technology

Side3. Construction Costs

Side4. Cost Reduction Method

......

Slot 4. Instructions

Side1. Case Analysis

\section{Similar Cases Retrieval}

Current traditional common retrieval method are the following: knowledge boot, nearest neighbor, inductive method. These methods are more suitable for retrieval of qualitative attributes, but not easy for retrieval of quantitative attributes. And it is even more difficult to be used for searching some fuzzy type of quantitative attributes [6].Gray correlation analysis method is a detailed analysis calculation method of the degree of similarity or correlation between the various factors in the whole system. The basic idea is to sort the factors according to the size of the correlation degree. [7].The use of weighted gray correlation coefficients is to reflect similarity of the new projects and known cases in the case database, a lower threshold is provided, when the weighted gray correlation coefficient is greater than the threshold, the case will be as the final retrieval results and used as a reference case of cost control.

Suppose $P$ is the set of construction project cases. The retrieved candidate case database is $P=\left(P_{1}, P_{2}, \cdots, P_{m}\right), \mathrm{m}$ represents the number of cases in the candidate case database. $P_{0}$ is a new project, it is to be a solved case.

Property features of case $P$ is expressed as $T=\left(T_{1}, T_{2}, \ldots, T_{\mathrm{n}}\right)$. The feature factor parameter of the $\mathrm{i}$-th reference work will be as a sub sequence, $X_{i}=\left\{X_{i}\left(T_{1}\right), X_{i}\left(T_{2}\right), \cdots \cdots, X_{i}\left(T_{n}\right)\right\}, i=1,2, \cdots, n$. The weight of the $i$-th property is $\omega_{i}, \omega_{i} \in(0,1), \Sigma \omega_{i}=1(i=1,2, \ldots, \mathrm{n}), \mathrm{n}$ is the total number of property features $(n \geq 1)$. Similarity search procedure is as follows: 
(1)Data Initialization.

After the primary search, messy data and qualitative data were needed to be normalized after quantization. In order to eliminate the impact of dimension and magnitude, the data is needed for non-dimensional treatment. Average method, the initial value method, the maximum method, the minimum value method, normalization method are used in the calculation methods. When using the average method for non-dimensional treatment, the formula is as follows:

$$
p_{i j}=\frac{x_{i j}}{\sum_{i=1}^{m} x_{i j} / m}
$$

(2) Calculation of Similarity.

The expression of the similarity of the weighted gray correlation coefficient is used to consider the characteristics of each feature. It can avoid the influence of association of each index on the cost, and make similarity calculation more accurate, intuitive and easy to compare. After normalization, the gray correlation coefficient is calculated, and the calculation steps are as follows:

1) After normalized treatment, sequence difference between each resulting subset $P_{i}$ and new construction $P_{o}$ in each characteristic factor can be obtained. The formula is as follows:

$$
\Delta T=\left|P_{O}(k)-P_{i}(k)\right| \quad k \in[1, n]
$$

2) Calculation of the minimum difference and the maximum difference is as follows:

$$
\begin{aligned}
& \Delta \min =\min _{i \in m} \min _{k \in n}\left(\left|p_{o}(k)-p_{i}(k)\right|\right) \\
& \Delta \max =\max _{i \in m} \max _{k \in n}\left(\left|p_{o}(k)-p_{i}(k)\right|\right)
\end{aligned}
$$

3) Calculation of Correlation Coefficient.

$$
\varepsilon_{i}(k)=r\left(p_{o}(k), p_{i}(k)\right)=\frac{\Delta \min +\theta_{\Delta} \max }{\Delta T+\theta_{\Delta} \max }
$$

In the above formula: $\varepsilon_{i}(k)$ is the correlation coefficient. It is the relative difference between the two curves on the point of the characteristic factors. $\theta$ is the resolution coefficient, $\theta \in(0,1)$, it is used to reduce the maximum levels between the two levels to avoid affecting the calculation of the overall coefficient. The smaller $\theta$ is, the greater the resolution capacity of the system is. On the contrary, it is smaller. Under normal circumstances it is 0.5 .

4) Weight Calculation

The weight of each attribute is the precondition of case retrieval. It reflects the importance of the property in relation to other attributes and the contribution of each feature attribute to solve the problem. There are many ways to calculate the weight. AHP, entropy, fuzzy clustering method are the most widely used methods [9]

5) Calculation of Correlation Degree.

Correlation degree is defined as $r_{i}, \omega_{i}(k)$ indicates that the characteristic factor $k$ is the weight of the construction cost. The calculation formula is as follows.

$$
r_{i}=\sum_{k=1}^{n} \omega_{i}(k) \varepsilon_{i}(k)
$$

6) The Lower Threshold Settings

Similarity can be directly used to judge the degree of similarity between the new construction and the case. But when we finally decide how to choose the case engineering, we need to set a threshold. When the similarity of the known case engineering and the new project is higher than the threshold, the case can be output as a higher similarity case to provide a reference for new construction.

The principles of setting the threshold are that the number of retrieved cases is as less as possible, and it is better to be associated with the new project as large as possible. 


\section{Summary}

In order to solve the problem of the low efficiency of the case knowledge description and the case retrieval in the field of the current construction cost control, knowledge representation, retrieval methods and the case database are applied to knowledge management. Then the construction enterprises can effectively reduce the impact of uncertainties, and improve the efficiency of cost control.

\section{Reference:}

[1] Song Xiaofei. A research on construction enterprises construction cost control [D]. Guangxi: Guangxi University, 2012.

[2] Alavi M, Leidner D. Knowledge management systems Issues, challenges, and benefits [J].Communications of the AIS, 1999, 1(7):2-28.

[3] Ou Lixiong, Song Chenying, Huang Kexin. A research on enterprises knowledge management method based on project oriented [J]. Science Technology and Engineering, 2006, 6 (4):507-510.

[4] Li Yajun. The study on the engineering cost estimation based on case-based reasoning [J]. Journal of Hebei University of Engineering, 2012, 30 (1):83-85.

[5] Xie Zhigang, Ren $\mathrm{Xu}$, Wei Zhensheng et al. Weapon fault diagnosis expert system based on case-based reasoning and fuzzy diagnosis [J]. Journal of Ordnance Engineering College, 2001, 13 (3):21-24.

[6] Chen Hong, Mao Hongbao. A new method based on rough set for case feature weight determination [J] . Journal of Xi'an Technological University, 2010, 30(4):402-405.

[7] Zhang Wenling, Jiang Shaohua, Liu Guodong. A research on reasoning model of engineering projects case based on grey relation analysis (J). Value Engineering, 2009 (11): 84-87.

[8] Lin Yi, Chen Mianyun, Liu Sifeng. Theory of grey systems: capturing uncertainties of grey information [J].Kybernetes, 2004, 33 (2):196-218. 